Provided for non-commercial research and education use. Not for reproduction, distribution or commercial use.

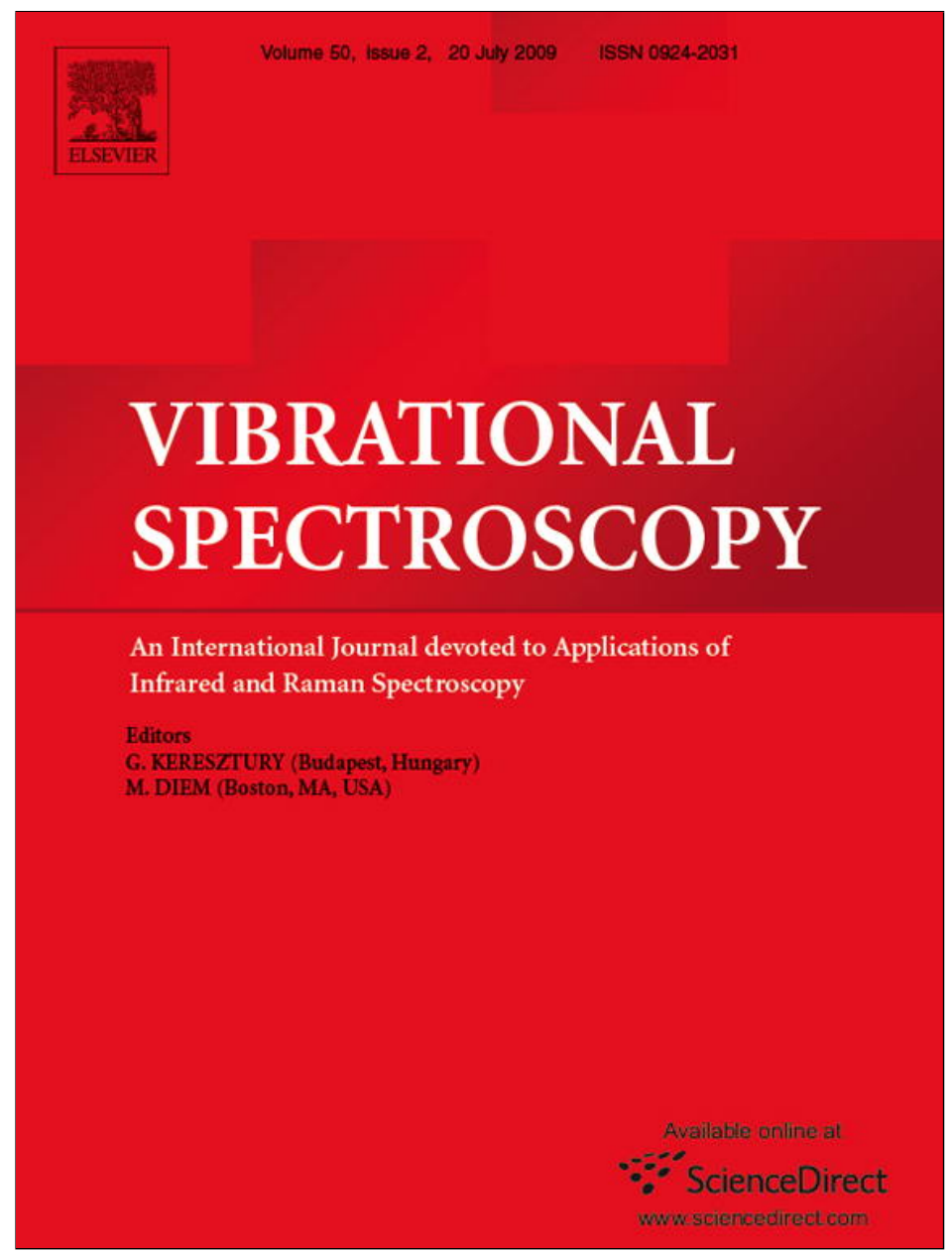

This article appeared in a journal published by Elsevier. The attached copy is furnished to the author for internal non-commercial research and education use, including for instruction at the authors institution and sharing with colleagues.

Other uses, including reproduction and distribution, or selling or licensing copies, or posting to personal, institutional or third party websites are prohibited.

In most cases authors are permitted to post their version of the article (e.g. in Word or Tex form) to their personal website or institutional repository. Authors requiring further information regarding Elsevier's archiving and manuscript policies are encouraged to visit:

http://www.elsevier.com/copyright 


\title{
Calculations of overtone NIR and NIR-VCD spectra in the local mode approximation: Camphor and Camphorquinone
}

\author{
Fabrizio Gangemi $^{\mathrm{a}, \mathrm{b}}$, Roberto Gangemi ${ }^{\mathrm{a}, \mathrm{b}}$, Giovanna Longhi ${ }^{\mathrm{a}, \mathrm{b}}$, Sergio Abbate ${ }^{\mathrm{a}, \mathrm{b}, *}$ \\ ${ }^{a}$ Dipartimento di Scienze Biomediche e Biotecnologie, Universita di Brescia, Viale Europa 11, 25123 Brescia, Italy

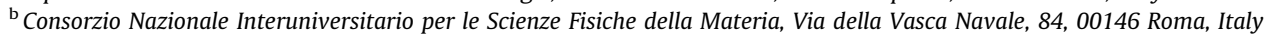

\section{A R T I C L E I N F O}

\section{Article history:}

Received 2 September 2008

Received in revised form 25 November 2008

Accepted 14 January 2009

Available online 23 January 2009

\section{Keywords:}

NIR

VCD

Local modes

Mechanical anharmonicity

Electrical anharmonicity

Morse potential

Camphor

Camphorquinone

\begin{abstract}
A B S T R A C T
We present a method to calculate near-infrared (NIR) and NIR-vibrational circular dichroism (NIR-VCD) spectra up to the second $\mathrm{CH}$-stretching overtone region in the local mode approximation. Atomic polar tensors and atomic axial tensors are first evaluated by DFT methodology for all $\mathrm{CH}$ stretching coordinates with systematic positive and negative displacements off-equilibrium and therefrom anharmonic dipole moment functions are constructed by polynomial interpolations. No adjustable parameters are employed up to this point. Rotational and dipole strengths are finally calculated by evaluating transition moments of Morse-type wave-functions. The method is applied to the case of Camphor and Camphorquinone, for which relevant differences in the vibrational circular dichroism (VCD) data are observed, which are predicted by our approach. Further steps are still to be made for a more complete treatment: the $a b$ initio evaluation of mechanical anharmonicity and the introduction of mechanical and electrical coupling between local modes.
\end{abstract}

() 2009 Elsevier B.V. All rights reserved.

\section{Introduction}

Several near-infrared (NIR) vibrational circular dichroism (VCD) spectra have been recently presented mainly from two research groups [1-9]. For the sake of historical exactness we must say that the first observation of NIR-VCD spectra was made by Keiderling and Stephens [10]. Besides some possible applications pointed out in Refs. [7,9], NIR-VCD data are interesting since they pose a challenge to interpretation and theory. As the mid-IR VCD case [11], also NIRVCD data can be really useful, NIR-VCD data can be made really useful, if they are preceded by the calculations of frequencies, dipole strengths and rotational strengths for vibrational transitions for $\mathrm{CH}$ stretching (or XH stretching in general) combination and overtone modes with minimal simplifying assumptions. This is a very hard task, the complexity increases with the number of $\mathrm{CH}$ bonds and the overtone order $[12,13]$. Another difficulty of NIR-VCD is the need to deal with anharmonicity in the force field and in the molecular dipole moment functions.

Model calculations [14,15], conducted under the rather restrictive assumption that rotational strengths are generated by

\footnotetext{
* Corresponding author at: Dipartimento di Scienze Biomediche e Biotecnologie, Universita di Brescia, Viale Europa 11, 25123 Brescia, Italy. Tel.: +39 030 3717415; fax: +39030 3701157

E-mail address: abbate@med.unibs.it (S. Abbate).
}

coupled electric bond dipole moments, have allowed us to get a qualitative idea of the aspect of fundamental IR- and overtone NIRVCD spectra at various overtone orders $\Delta v$ for a system of two coupled chirally disposed $\mathrm{CH}$-stretchings. The conclusion was that, except for rather high values of the interaction force constants between local stretchings, the NIR-VCD spectra, starting with the second overtone region, $\Delta v=3$, are too weak with respect to experimental findings, due to almost exact degeneracies of the pure overtone couplets of bands of opposite sign: we have interpreted this conclusion as a further validation of the local mode behavior. For this reason in Refs. [2-4] we simulated the NIR and NIR-VCD spectra at $\Delta v=3$ of Camphor and Camphor-related molecules or of 4-X-[2.2]paracyclophanes in the following way. We first calculated frequencies, dipole strengths and rotational strengths for all $\Delta v=1$ transitions associated with isolated $\mathrm{CH}$ stretching modes (the rest of the $\mathrm{CH}$ bonds being deuterated) in the harmonic approximation, as implemented in GAUSSIAN03 [16] at the DFT level. Then we corrected just the frequencies, by multiplying them by the given overtone order and by incorporating experimental mechanical anharmonicities, and transferred the rest of the results obtained for $\Delta v=1$ to the desired overtone order (in our case $\Delta v=3$ ). That is equivalent to assuming that the calculated local transitions be the only ones contributing to that region, i.e. assuming that there are no combination bands but just overtones of pure local modes. With that approach we could reproduce fairly well the general aspect of NIR absorption and NIR-VCD experi- 

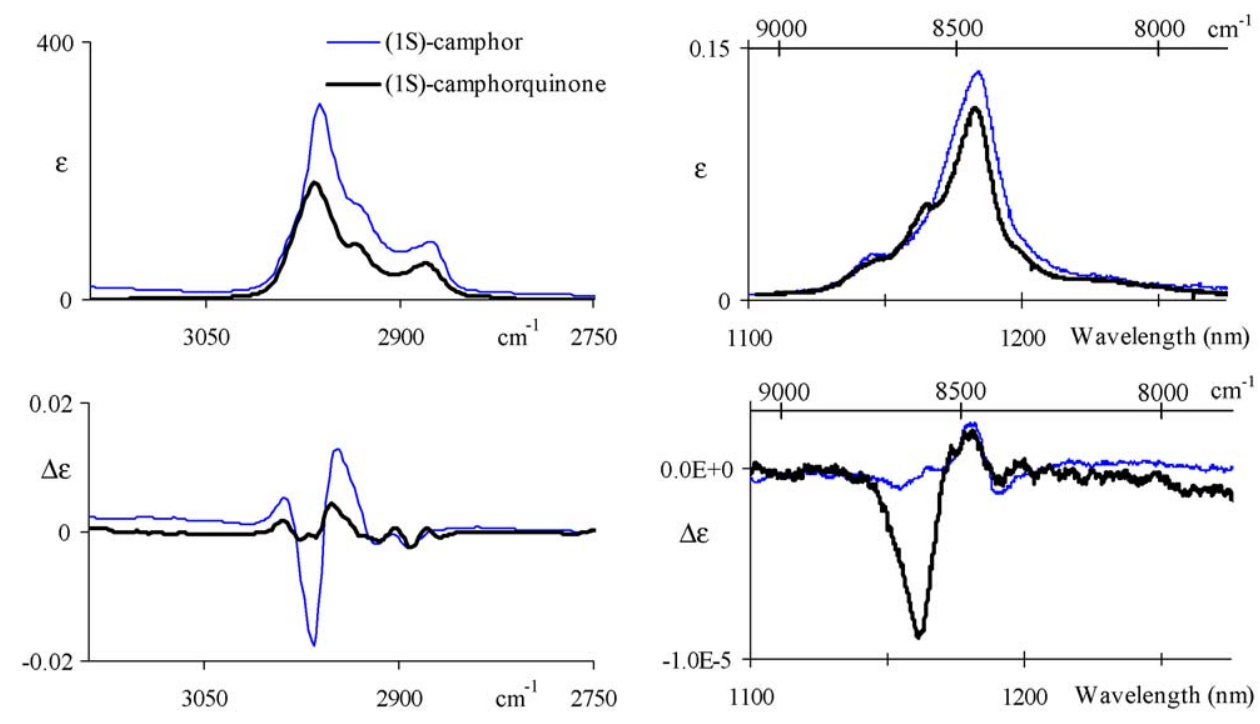

Panel 1A

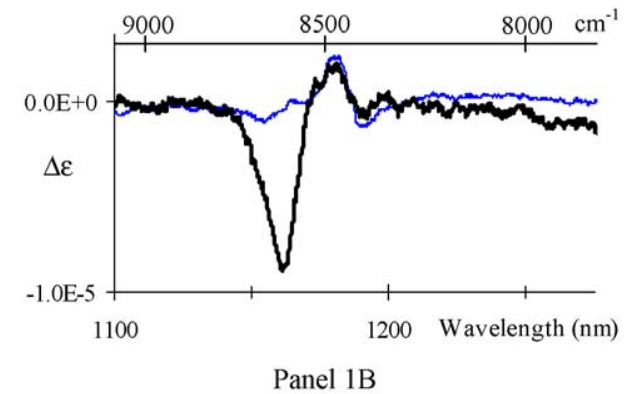

Fig. 1. Experimental IR absorption and IR-VCD spectra of (1S)-enantiomers of Camphor and Camphorquinone for $1 \mathrm{M} \mathrm{CCl}_{4}$ solutions (Panel $\left.1 \mathrm{~A}\right)(\Delta v=1)$; corresponding data for the NIR case in Panel 1B for $\Delta v=3$. For homogeneity of presentation of $\Delta v=1$ and 3 data, to the latter spectra a wavenumber scale axis has been added at the top; the original data were taken in linear wavelength scale.

mental spectra with the $y$-axis of the calculated spectra in arbitrary units. In the case of 4-X-[2.2]paracyclophanes [4] (see also Ref. [3]), we explicitly calculated transition moments for the Morse wavefunctions, and could provide absolute values also for the $y$-axis. Besides, in Ref. [4] we have evaluated some anharmonic terms in the APTs (atomic polar tensors) and thereby have significantly improved the calculations of NIR-VCD and NIR absorption spectra; however we did not consider there anharmonic corrections to the magnetic dipole moment terms (we notice, en passant, that the idea of using Morse local modes for interpreting NIR-VCD spectra had been suggested by Polavarapu [17] and is discussed also in Ref. [18]).

In this work we continue that study: we conduct systematic calculations of anharmonic electric and magnetic terms for all $\mathrm{CH}$ oscillators of rather large molecules, through the use of DFT calculations. We have chosen the interesting case of Camphor and Camphorquinone, experimental spectra of which had already been published by our group [1] and Nafie's group [8] independently for $\Delta v=3$ (we have shown in Fig. 1 the VCD and absorption $\operatorname{IR}(\Delta v=1)$ and $\operatorname{NIR}(\Delta v=3)$ spectra [1] of the (1S)-enantiomers). The reasons for our choice are two: (i) Camphor is one of the most studied molecules by $C D$ and VCD, one major motivation being that it is conformationally rigid and thus, even if the number of $\mathrm{CH}$-bond stretchings is large (16), their mutual orientation is fixed (see Fig. 2, where we reported the structure of (1S)-Camphor (a) and (1S)Camphorquinone (b)) and (ii) peculiar interesting differences appear in the VCD spectra of Camphor and Camphorquinone for the same absolute configuration of the stereogenic Carbon 1, which, in the first place, are a challenge for all theoretical models, and also may illuminate us on subtle physico-chemical differences between the two molecules. Indeed, at $\Delta v=1$ Camphorquinone spectra, especially the VCD one, are less intense than Camphor spectra, while at $\Delta v=3$ (NIR) one feature of Camphorquinone VCD spectrum is much larger than in Camphor; this feature is in correspondence with a shoulder in the absorption spectrum of Camphorquinone which is not present in Camphor's; the rest of the NIR-VCD and absorption spectra are almost the same for the two molecules.
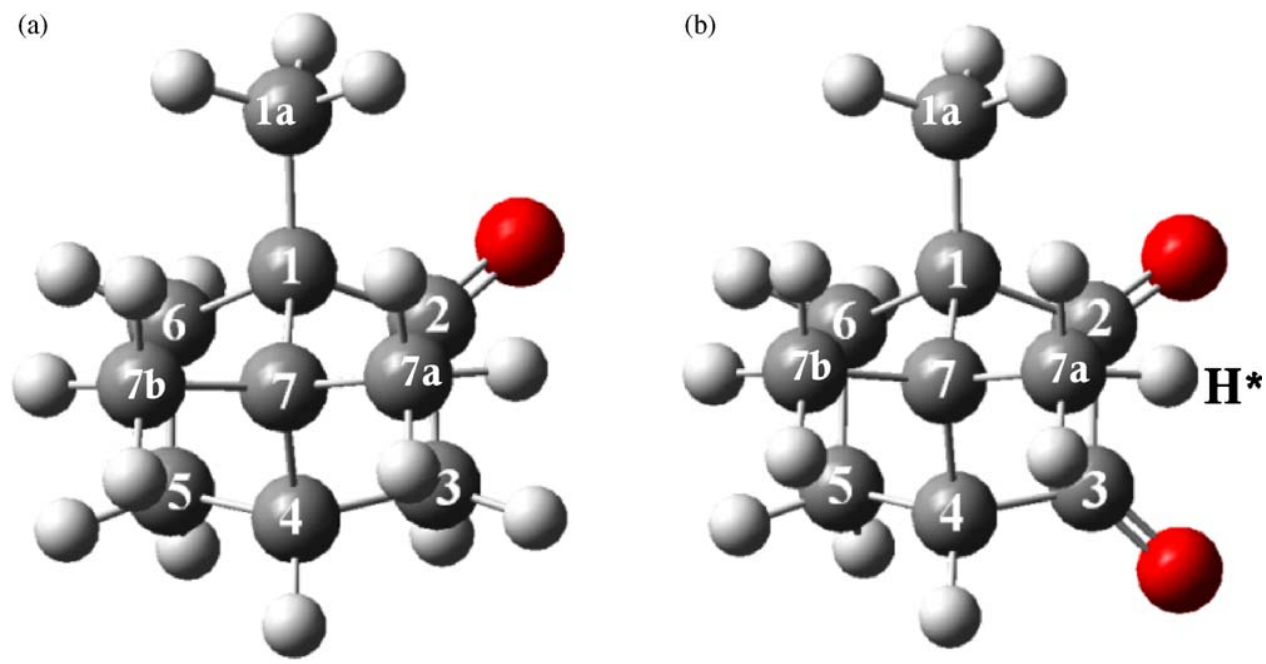

Fig. 2. Structure and group numbering of (1S)-Camphor (a) and (1S)-Camphorquinone (b) as obtained from Gaussview [16]. 

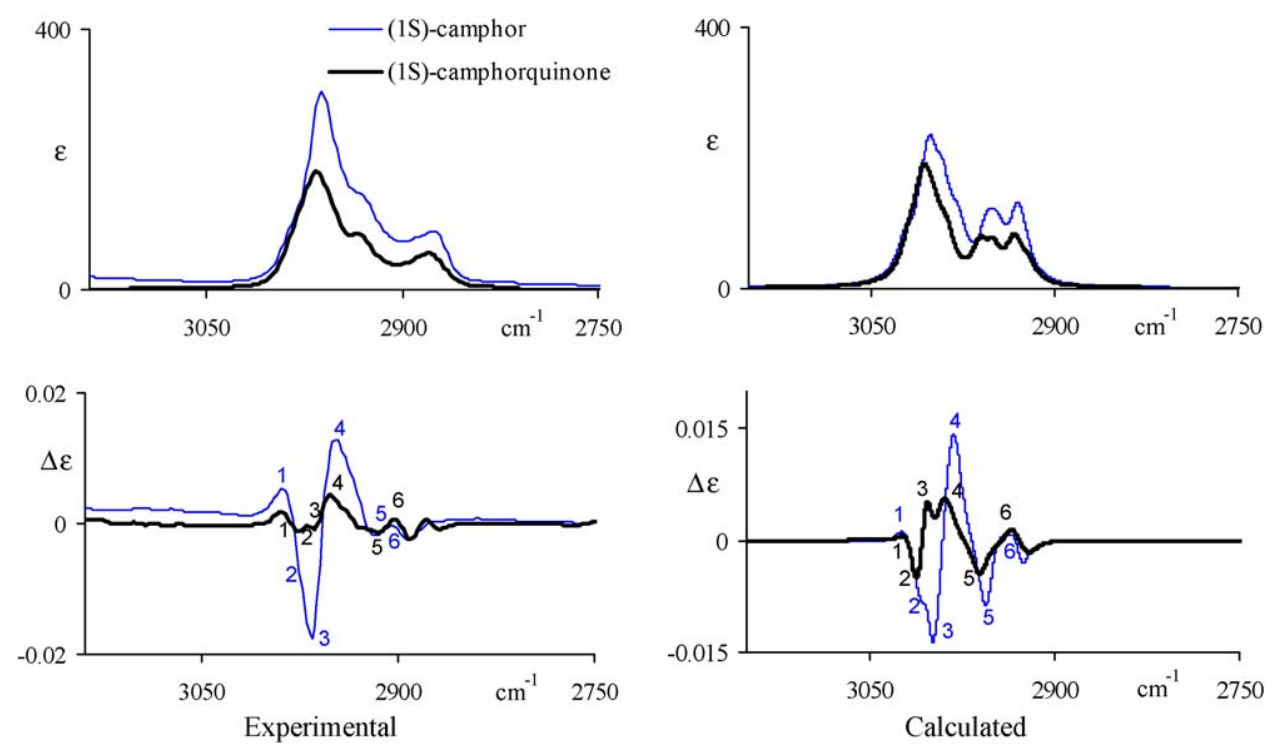

Fig. 3. Comparison of experimental and calculated IR and IR-VCD spectra of (1S)-Camphor and (1S)-Camphorquinone. The calculations are conducted by DFT methodology with Gaussian 03 [16] and finally shifted downward by $130 \mathrm{~cm}^{-1}(-2 \chi)$.

The interpretation of the IR and IR-VCD spectra at $\Delta v=1$ is straightforward, and is based on DFT (B3LYP/6-31G**) harmonic calculations modelled on the magnetic field perturbation method $[19,20]$. We present the comparison of experimental and calculated IR and IR-VCD spectra for the $\Delta v=1 \mathrm{CH}$-stretching region in Fig. 3. The agreement can be considered acceptable but not complete: the relative magnitude of IR and IR-VCD spectra for the two molecules is well accounted for, and the general aspect of sign alternation of VCD bands is partially accounted for. Analyzing the data of Fig. 3 from left to right we see that the first positive band is not adequately predicted; the Camphorquinone experimental VCD spectrum shows two negative bands labelled 2 and 3 in the figure, which are in correspondence with two calculated bands one of which is negative and one positive, the subsequent three bands are well predicted for the two molecules. We may generally state that the $\mathrm{CH}$ stretchings spectroscopic region is often not well predicted by standard normal mode calculations, due also to the presence of Fermi resonance. However we will not insist on these aspects, since this is not the focus of the present work. Indeed the aim of this work is to provide an interpretation of the second part of Fig. 1 ( $\Delta v=3$ spectra).

To achieve NIR-VCD spectra prediction, we have developed a method largely based on the work by Bak et al. [21], which was devised to account for perturbations brought about by anharmonicities in the IR region. This method is presented in Section 2. In Section 3 we comment on how actual calculations are performed, with particular attention to the derivation of electric and magnetic anharmonic terms from a polynomial fitting of DFT-derived results. In Section 4 we report the results of the calculations on the $\Delta v=3$ region of Camphor and Camphorquinone. The conclusions in Section 5 is devoted to a short discussion of our method and results, in view of what is needed to make the method completely free from ad hoc hypotheses.

\section{Method}

In this section we describe the method for the calculation of absorption and VCD spectra with the inclusion of electrical anharmonicity corrections and the subsequent restriction to the local-mode hypothesis.
Dipole and rotational strengths for a transition from the ground state 0 to an excited state $e$ are given by [22]

$D_{0 e}=\sum_{i=1}^{3}\left|\left\langle\mu_{i}\right\rangle_{0 e}\right|^{2}$

$R_{0 e}=\operatorname{Im} \sum_{i=1}^{3}\left\langle\mu_{i}\right\rangle_{0 e}\left\langle m_{i}\right\rangle_{e 0}$

where

$\left\langle\mu_{i}\right\rangle_{0 e}=\left\langle\psi_{0}\left|\hat{\mu}_{i}\right| \psi_{e}\right\rangle$

$\left\langle m_{i}\right\rangle_{e 0}=\left\langle\psi_{e}\left|\hat{m}_{i}\right| \psi_{0}\right\rangle$

and the operators $\hat{\mu}_{i}$ and $\hat{m}_{i}$ represent the $i$ th Cartesian component of the electric and magnetic dipole moment respectively.

In the Born-Oppenheimer (BO) approximation, a complete set of wave-functions for a molecule with $N$ atoms can be expressed in the form [23]

$\psi_{n v}(r, R)=\phi_{n}(r, R) \chi_{n v}(R)$

where $r$ is a shortened notation for electrons' coordinates, $R$ is for the corresponding nuclear ones, $\phi_{n}(r, R)$ is the electronic part of the wave-function, with index $n$ labeling the electronic levels, and $\chi_{n v}(R)$ is the nuclear part, with the additional index $v$ labeling the vibrational levels. (The rotational part of the wave-function is omitted here.)

If a transition from the ground state $\psi_{00}$ to a vibrational state $\psi_{0 v}$ is considered, it is well known that the matrix element of the electronic magnetic transition dipole moment vanishes and a nonvanishing contribution is obtained from the first perturbative correction to the BO approximation. The sum-over-states can be resolved with the help of the magnetic field perturbation method developed in Refs. $[19,20]$. The results of that approach are here briefly recalled mainly for notational purposes, as presented in Ref. [21]. The transition dipole moments can be expressed as

$\left\langle\mu_{i}\right\rangle_{0 v}=\left\langle\chi_{00}\left|\mu_{i}(R)\right| \chi_{0 v}\right\rangle$

$\left\langle m_{i}\right\rangle_{v 0}=\left\langle\chi_{0 v}\left|m_{i}(R)\right| \chi_{00}\right\rangle-\left\langle\chi_{00}\left|m_{i}(R)\right| \chi_{0 v}\right\rangle$ 
where

$$
\begin{aligned}
\mu_{i}(R)= & \left\langle\phi_{0}(R)\left|\hat{\mu}_{i}\right| \phi_{0}(R)\right\rangle \\
m_{i}(R)= & h \sum_{\alpha j} \frac{1}{M_{\alpha}} \\
& \times\left(\left\langle\frac{\partial \phi_{0}(R)}{\partial R_{\alpha j}} \mid \frac{\partial \phi_{0}(R)}{\partial B_{i}}\right\rangle_{B=0}+\frac{i}{4 h c} \sum_{k} \varepsilon_{i j k} R_{\alpha k} Z_{\alpha} e\right) \frac{h}{i} \frac{\partial}{\partial R_{\alpha j}} \\
= & h \sum_{\alpha j} \frac{1}{M_{\alpha}} A_{\alpha i j}(R) p_{\alpha j}
\end{aligned}
$$

where $\hbar$ is reduced Planck's constant, $h / 2 \pi, e$ is the electron charge, $c$ is the speed of light, $Z_{\alpha}$ is the atomic number and $A_{\alpha i j}$ is the atomic axial tensor (AAT) for the atom $\alpha, B_{i}$ is the $i$ th Cartesian component of the magnetic induction vector, and $M_{\alpha}$ is the $\alpha$-atom mass. The expressions above can be expanded around the equilibrium positions, $R_{\alpha i}^{0}$, of the nuclear coordinates,

$$
\begin{aligned}
\mu_{i}(R)= & \mu^{0}+\sum_{\alpha j}\left(\frac{\partial \mu_{i}}{\partial R_{\alpha j}}\right)_{0} r_{\alpha j}+\frac{1}{2} \sum_{\alpha j \beta k}\left(\frac{\partial^{2} \mu_{i}}{\partial R_{\alpha j} \partial R_{\beta k}}\right)_{0} r_{\alpha j} r_{\beta k} \\
& +\frac{1}{6} \sum_{\alpha j \beta k \gamma l}\left(\frac{\partial^{3} \mu_{i}}{\partial R_{\alpha j} \partial R_{\beta k} \partial R_{\gamma l}}\right)_{0} r_{\alpha j} r_{\beta k} r_{\gamma l}+\cdots \\
m_{i}(R)= & h \sum_{\alpha j} \frac{1}{M_{\alpha}}\left(A_{\alpha i j}^{0} p_{\alpha j}+\left(\frac{\partial A_{\alpha i j}}{\partial R_{\beta k}}\right)_{0} r_{\beta k} p_{\alpha j}+\right. \\
& \left.\frac{1}{2}\left(\frac{\partial^{2} A_{\alpha i j}}{\partial R_{\beta k} \partial R_{\gamma l}}\right)_{0} r_{\beta k} r_{\gamma l} p_{\alpha j}+\cdots\right)
\end{aligned}
$$

where $r_{\alpha j}=R_{\alpha i}-R_{\alpha i}^{0}$ are the displacements from the equilibrium coordinates and $p_{\alpha i}=(\hbar / i)\left(\partial / \partial R_{\alpha i}\right)$.

A slightly different expansion is given in Ref. [17] for the magnetic dipole moment, where the $r$ and $p$ variables appear in symmetric combinations, like $r p+p r$, and $r^{2} p+p r^{2}$. As already discussed in [17], if one takes into account the form of Eq. (7) and the commutation rules of operators $r$ and $p$, one can check that the more general expansion is equivalent to Eq. (11) as long as off-diagonal matrix elements are considered. Moreover, an additional term in $p^{3}$ is reported in the expansion of Ref. [17], which is not present in Eq. (11). Inclusion of that term in the present context would require an extension of the approach of Ref. [21] that is beyond the scope of this work.

When these expansions are substituted into Eqs.(6) and(7), taking into account that the first term in Eq. (10) gives no contribution, due to the orthogonality of the wave-functions, and assuming the wavefunctions to be real, the transition dipole moments become

$$
\begin{aligned}
\left\langle\mu_{i}\right\rangle_{0 v}= & \sum_{\alpha j} \Pi_{\alpha i j}^{0}\left\langle\chi_{00}\left|r_{\alpha j}\right| \chi_{0 v}\right\rangle \\
& +\frac{1}{2} \sum_{\alpha j \beta k}\left(\frac{\partial \Pi_{\alpha i j}}{\partial R_{\beta k}}\right)_{0}\left\langle\chi_{00}\left|r_{\alpha j} r_{\beta k}\right| \chi_{0 v}\right\rangle \\
& +\frac{1}{6} \sum_{\alpha j \beta k \gamma l}\left(\frac{\partial^{2} \Pi_{\alpha i j}}{\partial R_{\beta k} \partial R_{\gamma l}}\right)_{0}\left\langle\chi_{00}\left|r_{\alpha j} r_{\beta k} r_{\gamma l}\right| \chi_{0 v}\right\rangle+\cdots \\
\left\langle m_{i}\right\rangle_{v 0}= & 2 h \sum_{\alpha j} A_{\alpha i j}^{0} \frac{1}{M_{\alpha}}\left\langle\chi_{0 v}\left|p_{\alpha j}\right| \chi_{00}\right\rangle+2 h \sum_{\alpha j \beta k}\left(\frac{\partial A_{\alpha i j}}{\partial R_{\beta k}}\right)_{0} \frac{1}{M_{\alpha}} \\
& \times\left\langle\chi_{0 v}\left|r_{\beta k} p_{\alpha j}\right| \chi_{00}\right\rangle+\frac{h}{2} \sum_{\alpha j \beta k \gamma l}\left(\frac{\partial^{2} A_{\alpha i j}}{\partial R_{\beta k} \partial R_{\gamma l}}\right)_{0} \frac{1}{M_{\alpha}} \\
& \times\left(\left\langle\chi_{0 v}\left|r_{\beta k} r_{\gamma l} p_{\alpha j}\right| \chi_{00}\right\rangle-\left\langle\chi_{00}\left|r_{\beta k} r_{\gamma l} p_{\alpha j}\right| \chi_{0 v}\right\rangle\right)+\cdots
\end{aligned}
$$

where the APT $\Pi_{\alpha i j}=\partial \mu_{i} / \partial R_{\alpha j}$ has been introduced.
The solution of the mechanical problem, i.e. the determination of the nuclear wave-functions, provides us with the modes of vibration. As is usually done in the harmonic approximation, this is achieved by a transformation to mass-weighted variables

$r_{\alpha i}=\sum_{n} S_{\alpha i}^{n} Q_{n}$

$p_{\alpha i}=\sum_{n} M_{\alpha} S_{\alpha i}^{n} P_{n}$

where $P_{n}$ are the conjugate momenta of $Q_{n}$.

The same scheme may be used for the local-mode approach to be discussed below. After normalizing the coefficients $S_{\alpha j}^{n}$ through

$\frac{1}{m_{G}}=\sum_{\alpha j}\left(S_{\alpha j}^{n}\right)^{2}$

$L_{\alpha j}^{n}=\sqrt[\alpha j]{m_{G}} S_{\alpha j}^{n}$

where the normalization factor $m_{G}$ is a quantity with the dimensions of a mass, Eqs. (12) and (13) in the new variables, when restricted to one particular vibrational mode $n$, become

$$
\begin{aligned}
\left\langle\mu_{i}\right\rangle_{0 v}= & \sum_{\alpha j} \Pi_{\alpha i j}^{0} \frac{L_{\alpha j}^{n}}{\sqrt{m_{G}}}\left\langle 0\left|Q_{n}\right| v\right\rangle+\frac{1}{2} \sum_{\alpha j \beta k}\left(\frac{\partial \Pi_{\alpha i j}}{\partial R_{\beta k}}\right)_{0} \frac{L_{\alpha j}^{n} L_{\beta k}^{n}}{m_{G}} \\
& \times\left\langle 0\left|Q_{n}^{2}\right| v\right\rangle+\frac{1}{6} \sum_{\alpha j \beta k \gamma l}\left(\frac{\partial^{2} \Pi_{\alpha i j}}{\partial R_{\beta k} \partial R_{\gamma l}}\right)_{0} \frac{L_{\alpha j}^{n} L_{\beta k}^{n} L_{\gamma l}^{n}}{\left(m_{G}\right)^{3 / 2}}\left\langle 0\left|Q_{n}^{3}\right| v\right\rangle+\cdots
\end{aligned}
$$

$$
\begin{aligned}
\left\langle m_{i}\right\rangle_{v 0}= & 2 h \sum_{\alpha j} A_{\alpha i j}^{0} \frac{L_{\alpha j}^{n}}{\sqrt{m_{G}}}\left\langle v\left|P_{n}\right| 0\right\rangle \\
& +2 h \sum_{\alpha j} \sum_{\beta k}\left(\frac{\partial A_{\alpha i j}}{\partial R_{\beta k}}\right)_{0} \frac{L_{\alpha j}^{n} L_{\beta k}^{n}}{m_{G}}\left\langle v\left|Q_{n} P_{n}\right| 0\right\rangle \\
& +\frac{1}{2} h \sum_{\alpha j \beta k \gamma l}\left(\frac{\partial^{2} A_{\alpha i j}}{\partial R_{\beta k} \partial R_{\gamma l}}\right)_{0} \frac{L_{\alpha j}^{n} L_{\beta k}^{n} L_{\gamma l}^{n}}{\left(m_{G}\right)^{3 / 2}} \\
& \times\left(\left\langle v\left|Q_{n}^{2} P_{n}\right| 0\right\rangle-\left\langle 0\left|Q_{n}^{2} P_{n}\right| v\right\rangle\right)+\cdots
\end{aligned}
$$

The set of coefficients $L_{\alpha j}^{n}$ may be interpreted as the normalized Cartesian displacements of the $\alpha$-nucleus along the Cartesian axis $j$, corresponding to the vibrational mode $n$. In the hypothesis of a local $\mathrm{CH}$-stretching mode, these quantities are easily determined: to calculate the dipole and rotational strengths of each mode, the coordinate system may be chosen in such a way that the $z$-axis (which is numbered as 3 henceforth) is along the $\mathrm{CH}$ bond direction, and if the displacements of the two atoms from their equilibrium positions are called $z_{H}$ and $z_{C}$, the local mode coordinate can be written

$Q_{n}=\sqrt{m_{R}}\left(z_{H}-z_{C}\right)$

with $m_{R}=M_{H} M_{C} / M_{C H}$ and $M_{C H}=M_{H}+M_{C}$. The CH-stretching coordinate that can be defined from the instantaneous bond length $z$ as $z-z_{e}=z_{H}-z_{C}$ (where $z_{e}$ is the equilibrium value of the bond length), and its canonical conjugate $p$ are related to the variables $Q_{n}$ and $P_{n}$ through

$z-z_{e}=\frac{Q_{n}}{\sqrt{m_{R}}}$

$p=\sqrt{m_{R}} P_{n}$

The only non-vanishing coefficients $L_{\alpha j}^{n}$ are those with $\alpha=C$ or $H$, and with $j=3$, namely

$L_{H 3}^{n}=\frac{M_{C}}{\sqrt{M_{H}^{2}+M_{C}^{2}}}$ 
$L_{C 3}^{n}=-\frac{M_{H}}{\sqrt{M_{H}^{2}+M_{C}^{2}}}$

and the normalization factor $m_{G}$ for this mode can be expressed in terms of the reduced mass $m_{R}$ as

$m_{G}=m_{R} \frac{M_{C H}^{2}}{M_{H}^{2}+M_{C}^{2}}$

After substitution of the variable $z$ in place of $Q_{n}$ in Eqs. (18) and (19), one can observe that the differential operators (as expected) have the form

$$
\sum_{\alpha=H, C} L_{\alpha 3}^{n} \sqrt{\frac{m_{R}}{m_{G}}} \frac{\partial}{\partial R_{\alpha 3}}=\frac{M_{C}}{M_{C H}} \frac{\partial}{\partial R_{H 3}}-\frac{M_{H}}{M_{C H}} \frac{\partial}{\partial R_{C 3}}=\frac{\partial}{\partial z}
$$

so that the transition dipole moments are given by

$$
\begin{aligned}
\left\langle\mu_{i}\right\rangle_{0 v}= & \sum_{\alpha=H, C} \Pi_{\alpha i 3}^{0} L_{\alpha 3}^{n} \sqrt{\frac{m_{R}}{m_{G}}}\left\langle 0\left|z-z_{e}\right| v\right\rangle \\
& +\frac{1}{2} \sum_{\alpha=H, C}\left(\frac{\partial \Pi_{\alpha i 3}}{\partial z}\right)_{0} L_{\alpha 3}^{n} \sqrt{\frac{m_{R}}{m_{G}}}\left\langle 0\left|\left(z-z_{e}\right)^{2}\right| v\right\rangle \\
& +\frac{1}{6} \sum_{\alpha=H, C}\left(\frac{\partial^{2} \Pi_{\alpha i 3}}{\partial z^{2}}\right)_{0} L_{\alpha 3}^{n} \sqrt{\frac{m_{R}}{m_{G}}}\left\langle 0\left|\left(z-z_{e}\right)^{3}\right| v\right\rangle+\cdots
\end{aligned}
$$

$$
\begin{aligned}
\left\langle m_{i}\right\rangle_{\nu 0}= & 2 h \sum_{\alpha=H, C} A_{\alpha i 3}^{0} \frac{L_{\alpha 3}^{n}}{\sqrt{m_{R} m_{G}}}\langle v|p| 0\rangle \\
& +2 h \sum_{\alpha=H, C}\left(\frac{\partial A_{\alpha i 3}}{\partial z}\right)_{0} \frac{L_{\alpha 3}^{n}}{\sqrt{m_{R} m_{G}}}\left\langle v\left|\left(z-z_{e}\right) p\right| 0\right\rangle \\
& +\frac{1}{2} h \sum_{\alpha=H, C}\left(\frac{\partial^{2} A_{\alpha i 3}}{\partial z^{2}}\right)_{0} \frac{L_{\alpha 3}^{n}}{\sqrt{m_{R} m_{G}}} \\
& \times\left(\left\langle v\left|\left(z-z_{e}\right)^{2} p\right| 0\right\rangle-\left\langle 0\left|\left(z-z_{e}\right)^{2} p\right| v\right\rangle\right)+\cdots
\end{aligned}
$$

As it has been discussed by various authors [19,21,24], atomic polar and atomic axial tensors depend on the choice of the coordinate system, and special care is needed in their use: APTs depend on axes' orientation, but are origin-independent for a neutral molecule, while AATs depend on both the origin and the axes' orientation of the coordinate system. However in principle rotational and dipole strengths, which are observable quantities, do not depend on the choice of the reference frame. In Ref. [21] it was shown indeed that the use of electric and magnetic dipole moment operators as those given in Eqs. (8) and (9) above gives rise to origin-independent rotational strengths, provided that AATs have the correct origin-dependence. This, in turn, is ensured [24] by the use of GIAO atomic orbitals, as implemented e.g. in the Gaussian 03 VCD calculation procedure employed here (see Section 3). In Eqs. (27) and (28), one local CH stretching mode is considered at a time, so that one is free to choose the most convenient reference frame, as long as all the terms appearing in the expressions are calculated in the same system.

As described in the next subsection, the derivatives of atomic polar and axial tensors are calculated $a b$ initio, taking into account Eqs. (23) and (24) to determine the magnitude of atomic displacements to be used in the numerical procedure.

In addition, in order to implement the above-described computational method, frequencies and matrix elements must be determined: we assume as mechanical harmonic frequencies, $\omega_{0 n}$, those obtained by a standard harmonic calculation with selective deuteration [2-4] and the correct values for overtones are calculated by including the anharmonicity parameter $\chi_{n}$ in the expression of vibrational energy eigenvalues

$E_{n v}=h c\left(\omega_{0 n}\left(v+\frac{1}{2}\right)-\chi_{n}\left(v+\frac{1}{2}\right)^{2}\right)$

As previously done [2-4], the same value $\chi_{n}=65 \mathrm{~cm}^{-1}$, taken from the analysis of experimental overtone absorption spectra, is assumed for every local mode $n$ (i.e. for every $\mathrm{CH}$ group) and is applied to both (1S)-Camphor and (1S)-Camphorquinone.

The matrix elements in Eqs. (27) and (28) are calculated with Morse wave-functions, assuming the following Morse potential for local mode $n$ [25]:

$U_{n}(z)=D_{n}\left(1-\mathrm{e}^{a_{n}\left(z-z_{e}\right)}\right)^{2}$

$a_{n}=\sqrt{\frac{8 \pi^{2} m_{R} c \chi_{n}}{h}}$

$D_{n}=\frac{\omega_{0 n}^{2}}{4 \chi_{n}}$

Details about matrix elements' evaluations with Morse wavefunctions are described in Appendix A.

\section{Numerical calculations}

\subsection{DFT calculations}

In order to derive the APT and AAT derivatives appearing in Eqs. (27) and (28), we have first studied $a b$ initio the APTs and AATs at different values of $\mathrm{CH}$ bond lengths; this approach had previously been applied by Kjaergaard and Henry [26,27] for studying electric dipole moments. From the continuous curve constructed by polynomial interpolation, we finally obtained its derivatives at equilibrium.

Calculations were performed by the Gaussian 03 suite of programs [16], first optimizing with the DFT method the molecule geometry with a $6-31 \mathrm{G}^{* *}$ basis set and the B3LYP functional. Then a number of modified input geometries corresponding to the different stretching displacements were generated starting from the equilibrium optimized geometry; ten points for each local mode were verified to be enough, as explained below. For each $\mathrm{CH}-$ bond stretching mode, Cartesian axes have been chosen such that the $z$-axis lies along the considered bond. As discussed in Section 2, this procedure is correct as long as all the terms involved in the calculation of the dipole and rotational strength of a given local mode are referred to the same Cartesian axes system: indeed all the points corresponding to displacements of one $\mathrm{CH}$ group are generated here in the same coordinate system, and the results from Gaussian 03 are always taken in the input coordinate system.

\subsection{Derivation of anharmonic terms for the APTs and AATs}

In the present treatment each $\mathrm{CH}$-bond stretching local mode is supposed to occur as if the two atoms were isolated from the rest of the molecule, and their mass-center fixed at its rest position. Thus the Hydrogen and Carbon atoms' motions bear a relationship to each other described by Eqs. (23) and (24) and we consider their APT and AAT components as functions of their relative distance.

In principle though, and as done in GAUSSIAN03 for the harmonic case, one might regard as a more general approach to calculate the derivatives with respect to the Cartesian coordinates of each single atom and to combine them later according to the actual modes of vibration. However, even in the hypothesis of local $\mathrm{CH}$ stretching modes, this would require evaluation of AATs and APTs on a two-dimensional grid of points to work out mixed derivatives that appear in second-order corrections (see Eqs. (18) 
and (19)). On the other hand, restriction to derivatives with respect to Hydrogen atoms alone, as practised in our previous paper [4], is in general inaccurate, as discussed in Section 4 of the present work.

The method adopted here, instead, evaluates also second-order (and in principle higher order) corrections by a one-dimensional grid, with relevant reduction of the computing time: as the Hydrogen atom is displaced from equilibrium in the interval ( $-0.4 \AA, 0.4 \AA$ ) by steps of $0.08 \AA$, the corresponding Carbon atom is also displaced and its displacement is calculated according to Eqs. (23) and (24).

The maximum Hydrogen atom compression $(-0.4 \AA)$, which might seem overrated, is justified a posteriori, in that the interpolated curves show no special (pathological) behaviour about this end, but continue smooth. As to the stepsize for Hydrogens, a spacing of $0.02 \AA$ was initially employed, but by trying in a few cases we have concluded that, adopting the value $0.08 \AA$ does not significantly affect the values of derivatives at equilibrium.

The data thus collected are arranged to represent the dependence of APT and AAT on local stretching coordinates. We express each component as an eight-degree polynomial in the scanned coordinate using a linear regression routine. By trying polynomials with increasing degrees starting from four, we have observed that the first and second derivatives of APTs and AATs vary considerably with the degree, whereas they become steady from degree seven/eight upwards. On the other hand the number of points makes it meaningless to use polynomials of higher degree.

\section{Results}

In Fig. 4 we report the results of interpolations for Hydrogen APTs and AATs for (1S)-Camphor (a) and (1S)-Camphorquinone (b). Of course, since the stretching local mode is along the bond direction, we concentrate just on the three tensor components containing the derivative with respect to $z$. While $z$ has always a univocal definition, $x$ and $y$ are somewhat arbitrary, so that it seems proper to consider in Fig. 4 (not in actual calculations, where $x$ and $y$ are treated distinctly!), a direction accounting for the components transverse to $z$ jointly, viz. quadratically summed (henceforth "transverse component").

One may notice that the $z$-component of APTs and their derivatives are one order of magnitude larger than the corresponding transverse components, while the opposite is true for AAT. This is intuitively acceptable, since stretching $\mathrm{CH}$ bonds give rise to larger variation of polarity on the $\mathrm{CH}$ bonds and thus to larger $z$ components in the electric dipole moment, while the same charge rearrangement generates larger magnetic dipole moment perpendicular to the $\mathrm{CH}$ bond. Since $x$ and $y$ components of AAT are comparatively large, also $x$ and $y$ components of APT should not be neglected, even if small, since the dot product of APT and AAT is needed in calculating rotational strengths, unlike what we had done earlier [2-4]. To make our considerations more quantitative, we give in Table 1 the values of APT and AAT at $z=0$ and their first derivatives with respect to $z$ still at $z=0$. We observe that APT $z$ components (longitudinal components) have a negative value and a negative derivative in all cases, and this is in accord with the results of Ref. [26] and with what found from the interpretation of NIR-absorption spectra [28] (see also Ref. [4]). Indeed, one obtains for $(\partial / \partial z) \Pi_{\mathrm{Hzz}}$ an average value of $-0.53 \mathrm{e} / \AA$ for Camphor and $-0.50 \mathrm{e} / \mathrm{A}$ for Camphorquinone, values which compare very well with the values of $-0.547 \mathrm{e} / \AA$ and $-0.485 \mathrm{e} / \AA$ determined experimentally for $\left(\partial^{2} \mu / \partial r^{2}\right)$ of equatorial and axial $\mathrm{CH}$ bonds respectively in dioxane [28]. Also, from Table 1 , we gather an average value for $\Pi_{\mathrm{Hzz}}$ at $z=0$ of -0.149 e for Camphor and of -0.137 e for Camphorquinone, that compares quite well with the experimental value of $-0.195 \mathrm{e}$ and $-0.143 \mathrm{e}$ for $(\partial \mu / \partial r)$ of equatorial and axial $\mathrm{CHs}$ respectively in dioxane [28]. The success of the DFT method in predicting electric anharmonic terms had been already observed in Ref. [26] for the $\mathrm{CH}$ bonds of cyclohexane and also for aromatic CHs in paracyclophanes [4]. No clear analysis and meaningful comparison was made for AAT values at $z=0$ or their first derivatives, but we report them anyway in Table 1 , since they show a trend. Finally, it is interesting to notice in Fig. 4 that a curve is clearly distinct from the others in the transverse components of APTs and AATs, namely the one corresponding to the single Hydrogen atom (C4-H) (also $\mathrm{C} 7 \mathrm{a}-\mathrm{H}^{*}$ of Camphorquinone, see Fig. 2, has a distinct behaviour for just transverse components of AAT). The reason for this peculiarity we may guess is due to its position in the molecule, this $\mathrm{CH}$ bond being under strong influence from the neighbouring oxygen atoms. The effect is larger for (1S)-Camphorquinone.

In Fig. 5 calculations of absorption and CD spectra in the NIR region are reported for (1S)-Camphor and (1S)-Camphorquinone at various levels of approximation. The spectra are obtained by applying a Lorentzian line-shape to each transition centered at the calculated frequencies, with areas equal to the calculated dipole strengths for absorption and rotational strengths for VCD, each one with a $\Delta \lambda \approx 6 \mathrm{~nm}$ bandwidth. The reason for this choice is that it best reproduces the results of NIR-VCD (vide infra); actually it is close also to the value employed in Ref. [4]. The lines labeled 'Abs0' and 'CDO' only include the lowest contributions, corresponding to the very first terms of Eqs. (18) and (19), hence they do not include electrical anharmonicity at all. The lines labeled 'Abs11' and 'CD11' include only Hydrogen-atom contributions from the first anharmonic terms of Eqs. (18) and (19), i.e. terms with both $\alpha$ and $\beta$ therein corresponding to the Hydrogen atom in the $\mathrm{CH}$ bond stretching under observation; the lines labeled 'Abs12' and ' $\mathrm{CD} 12$ ' include also first-order anharmonic contributions with $\alpha=\beta=C$, and the lines 'Abs13' and 'CD13' contain the full first-order corrections, including mixed $\mathrm{C}-\mathrm{H}$ terms that are ignored in the previous ones (i.e. $\alpha=\mathrm{H}, \beta=\mathrm{C}$, and $\alpha=\mathrm{C}, \beta=\mathrm{H}$ ). Finally, the lines 'Abs2' and 'CD2' contain corrections up to second-order, calculated according to Eqs. (27) and (28), where the derivatives of the APT's and AAT's are determined with respect to the internal $\mathrm{CH}$-stretching variable and naturally include all Hydrogen and Carbon atoms' contributions with proper weights. Comparison of the different firstorder results shows that restricting to Hydrogen atoms' contributions leads to significant errors and, among the neglected terms, the most important ones are mixed $\mathrm{C}-\mathrm{H}$ contributions. As discussed in Section 2, this leads to the conclusion that the best approach is to scan the APT's and AAT's along the internal CH-stretching variable, as is done for obtaining the results of Fig. 5 called 'Abs2' and 'CD2'.

As a general rule one knows that in order to best reproduce spectra at $\Delta v=2$ one needs to consider anharmonic terms relative to $z^{2}$ terms, for $\Delta v=3$ one needs to consider terms of the order $z^{3}$ and so forth $[26,28,29]$. In this case one may notice that the secondorder corrections (relative to $z^{3}$ ) are smaller than the first-order ones (relative to $z^{2}$ ) and generally have opposite sign. Their use does not significally change the results except in the case of NIR-VCD spectra of (1S)-Camphor.

To let the reader further appreciate the relative importance of the various terms, we give in Table 2 the calculated wavelengths $\lambda(\mathrm{nm})$, dipole strengths $D\left(\times 10^{-42} \mathrm{esu}^{2} \mathrm{~cm}^{2}\right)$ and rotational strengths $R\left(\times 10^{-47} \mathrm{esu}^{2} \mathrm{~cm}^{2}\right)$ at the three levels of approximations 0,13 , and 2 described above. One may notice from this table that VCD and absorption spectra are originated by closely lying transitions. This is a consequence of the fact that the calculated DFT harmonic frequencies of the isolated $\mathrm{CHs}$ are quite similar and this is ultimately related to the peculiar cage-type structure of Camphor as pointed out in Ref. [3]. The correct sign of the calculated VCD bands, giving rise to the alternating features that match the observed ones, is related to the proper calculation of the rotational strengths 
(a)

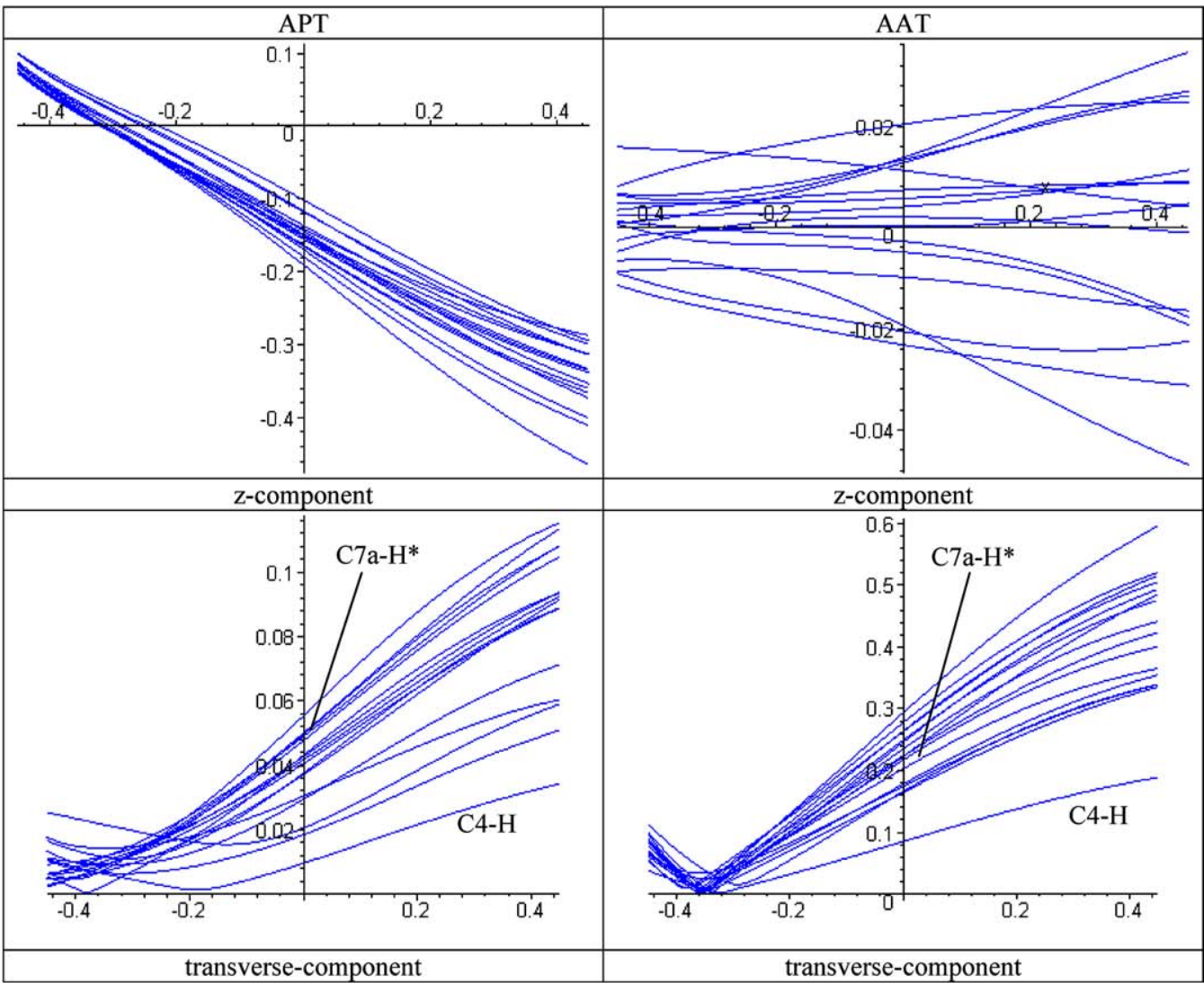

(b)

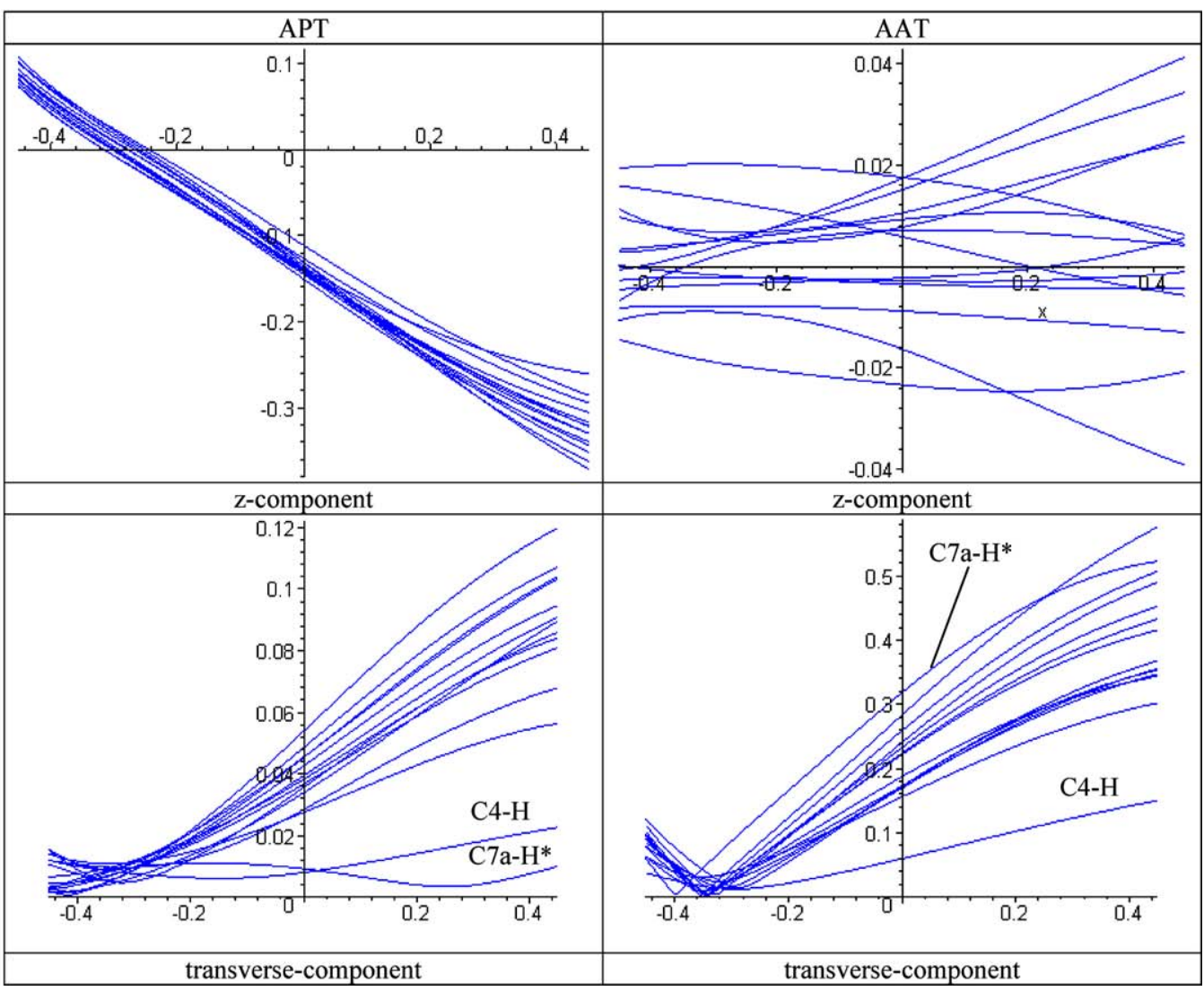

Fig. 4. Dependences of longitudinal and transverse components of APTs and AATs of Hydrogen atoms for (1S)-Camphor (a) and (1S)-Camphorquinone (b) on corresponding $\mathrm{CH}$-bond lengths' displacements ( $z$-directions) (see text). By definition, APT and AAT transverse components are always positive and this requires attention in looking at the results in the figure. APT's are in units of e (electrons), AAT's are in units of $\left(e a_{0}\right) /(\hbar c)$, where $a_{0}$ is the Bohr radius, and $c$ the velocity of light. On $x$-axes, displacements are in angstroms $(\AA ̊ ㇒)$. 
Table 1

APT and AAT values (upper half) and their first-order derivatives (lower half) for (1S)-Camphor and (1S)-Camphorquinone at equilibrium position.

\begin{tabular}{|c|c|c|c|c|c|c|c|c|}
\hline & \multicolumn{2}{|c|}{ Camphor APT } & \multicolumn{2}{|c|}{ Camphor AAT } & \multicolumn{2}{|c|}{ Camphorquinone APT } & \multicolumn{2}{|c|}{ Camphorquinone AAT } \\
\hline & $\Pi_{H t z}$ & $\Pi_{\mathrm{Hzz}}$ & $A_{H t z}$ & $A_{\mathrm{Hzz}}$ & $\Pi_{H t z}$ & $\Pi_{\mathrm{Hzz}}$ & $A_{H t z}$ & $A_{H z z}$ \\
\hline \multicolumn{9}{|c|}{ APT's and AAT's at equilibrium position } \\
\hline C1a-H & 0.042 & -0.137 & 0.246 & -0.010 & 0.038 & -0.127 & 0.240 & -0.009 \\
\hline $\mathrm{C} 1 \mathrm{a}-\mathrm{H}$ & 0.037 & -0.150 & 0.235 & 0.013 & 0.034 & -0.140 & 0.232 & 0.015 \\
\hline $\mathrm{C} 1 \mathrm{a}-\mathrm{H}$ & 0.049 & -0.118 & 0.166 & 0.011 & 0.046 & -0.111 & 0.172 & 0.006 \\
\hline $\mathrm{C} 3-\mathrm{H}$ & 0.021 & -0.102 & 0.223 & -0.023 & - & - & - & - \\
\hline $\mathrm{C} 3-\mathrm{H}$ & 0.037 & -0.116 & 0.165 & 0.007 & - & - & - & - \\
\hline $\mathrm{C} 4-\mathrm{H}$ & 0.010 & -0.190 & 0.085 & 0.004 & 0.008 & -0.131 & 0.060 & 0.017 \\
\hline $\mathrm{C} 5-\mathrm{H}$ & 0.055 & -0.179 & 0.224 & 0.020 & 0.054 & -0.135 & 0.172 & 0.018 \\
\hline $\mathrm{C} 5-\mathrm{H}$ & 0.043 & -0.169 & 0.262 & 0.006 & 0.036 & -0.138 & 0.173 & -0.002 \\
\hline C6-H & 0.050 & -0.144 & 0.178 & -0.021 & 0.049 & -0.131 & 0.169 & -0.023 \\
\hline $\mathrm{C} 6-\mathrm{H}$ & 0.029 & -0.149 & 0.174 & -0.003 & 0.028 & -0.138 & 0.156 & 0.009 \\
\hline$C 7 a-H$ & 0.048 & -0.163 & 0.202 & 0.013 & 0.043 & -0.145 & 0.187 & 0.011 \\
\hline $\mathrm{C} 7 a-\mathrm{H}$ & 0.041 & -0.153 & 0.249 & -0.019 & 0.037 & -0.142 & 0.224 & -0.016 \\
\hline$C 7 a-H^{*}$ & 0.018 & -0.142 & 0.277 & -0.005 & 0.009 & -0.137 & 0.318 & -0.003 \\
\hline$C 7 b-H$ & 0.031 & -0.149 & 0.293 & 0.000 & 0.028 & -0.144 & 0.284 & -0.003 \\
\hline$C 7 b-H$ & 0.043 & -0.155 & 0.264 & 0.014 & 0.039 & -0.143 & 0.259 & 0.008 \\
\hline \multirow[t]{3}{*}{$C 7 b-H$} & 0.050 & -0.165 & 0.217 & 0.002 & 0.045 & -0.150 & 0.222 & 0.007 \\
\hline & \multicolumn{2}{|c|}{ Camphor APT } & \multicolumn{2}{|c|}{ Camphor AAT } & \multicolumn{2}{|c|}{ Camphorquinone APT } & \multicolumn{2}{|c|}{ Camphorquinone AAT } \\
\hline & $\left(\partial_{z} \Pi_{H i z}\right)_{t}$ & $\partial_{z} \Pi_{\mathrm{Hzz}}$ & $\left(\partial_{z} A_{H i z}\right)_{t}$ & $\partial_{z} A_{H z z}$ & $\left(\partial_{z} \Pi_{H i z}\right)_{t}$ & $\partial_{z} \Pi_{\mathrm{Hzz}}$ & $\left(\partial_{z} A_{H i z}\right)_{t}$ & $\partial_{z} A_{H z z}$ \\
\hline \multicolumn{9}{|c|}{ APT's and AAT's first-order derivatives at equilibrium position } \\
\hline C1a-H & 0.118 & -0.490 & 0.646 & 0.037 & 0.117 & -0.478 & 0.663 & 0.043 \\
\hline $\mathrm{C} 1 \mathrm{a}-\mathrm{H}$ & 0.117 & -0.483 & 0.716 & -0.010 & 0.112 & -0.464 & 0.728 & -0.007 \\
\hline $\mathrm{C} 1 \mathrm{a}-\mathrm{H}$ & 0.139 & -0.482 & 0.542 & -0.016 & 0.137 & -0.470 & 0.571 & -0.029 \\
\hline $\mathrm{C} 3-\mathrm{H}$ & 0.109 & -0.481 & 0.662 & -0.021 & - & - & - & - \\
\hline $\mathrm{C} 3-\mathrm{H}$ & 0.139 & -0.506 & 0.473 & 0.006 & - & - & - & - \\
\hline $\mathrm{C} 4-\mathrm{H}$ & 0.058 & -0.676 & 0.272 & 0.009 & 0.038 & -0.570 & 0.216 & 0.050 \\
\hline $\mathrm{C} 5-\mathrm{H}$ & 0.168 & -0.613 & 0.571 & 0.019 & 0.174 & -0.551 & 0.560 & -0.015 \\
\hline $\mathrm{C} 5-\mathrm{H}$ & 0.142 & -0.599 & 0.686 & 0.006 & 0.132 & -0.568 & 0.496 & 0.005 \\
\hline C6-H & 0.153 & -0.546 & 0.510 & -0.022 & 0.154 & -0.524 & 0.536 & -0.012 \\
\hline C6-H & 0.111 & -0.559 & 0.476 & -0.012 & 0.110 & -0.538 & 0.434 & 0.013 \\
\hline$C 7 a-H$ & 0.139 & -0.538 & 0.521 & 0.031 & 0.131 & -0.511 & 0.510 & 0.026 \\
\hline $\mathrm{C} 7 a-H$ & 0.117 & -0.502 & 0.680 & -0.061 & 0.110 & -0.488 & 0.630 & -0.045 \\
\hline$C 7 a-H^{*}$ & 0.067 & -0.442 & 0.730 & -0.012 & 0.023 & -0.416 & 0.726 & -0.005 \\
\hline$C 7 b-H$ & 0.077 & -0.461 & 0.811 & -0.001 & 0.075 & -0.458 & 0.799 & -0.003 \\
\hline$C 7 b-H$ & 0.123 & -0.506 & 0.720 & 0.043 & 0.123 & -0.491 & 0.731 & 0.027 \\
\hline$C 7 b-H$ & 0.149 & -0.541 & 0.578 & -0.001 & 0.144 & -0.517 & 0.611 & 0.003 \\
\hline
\end{tabular}

Data for hydrogen atoms of corresponding positions in the two compounds are presented in a row (the parallel is supported by the similarity of structures in Fig. 2a and $b$ ). Column headings: $\Pi=$ APT, $A=$ AAT, $\partial_{z}=\partial / \partial z$; as to indexes: $\Pi_{\alpha i j}=\partial \mu_{i} / \partial R_{\alpha j}$, where $\alpha$ is the stretched atom $\Pi_{H t z}=\sqrt{\Pi_{H x z}^{2}+\Pi_{H y z}^{2}} ;\left(\partial_{z} \Pi_{H i z}\right)_{t}=\sqrt{\left(\partial_{z} \Pi_{H x z}\right)^{2}+\left(\partial_{z} \Pi_{H y z}\right)^{2}}$, and analogously for $A$. Side column on the left associates to each row the chemical group to which the stretched atom belongs. Units of measure are (e) for $\Pi$ 's and ( $\left.e a_{0}\right) /(\hbar c)$ for $A$ 's. Derivatives are with respect to lengths in $\AA$.
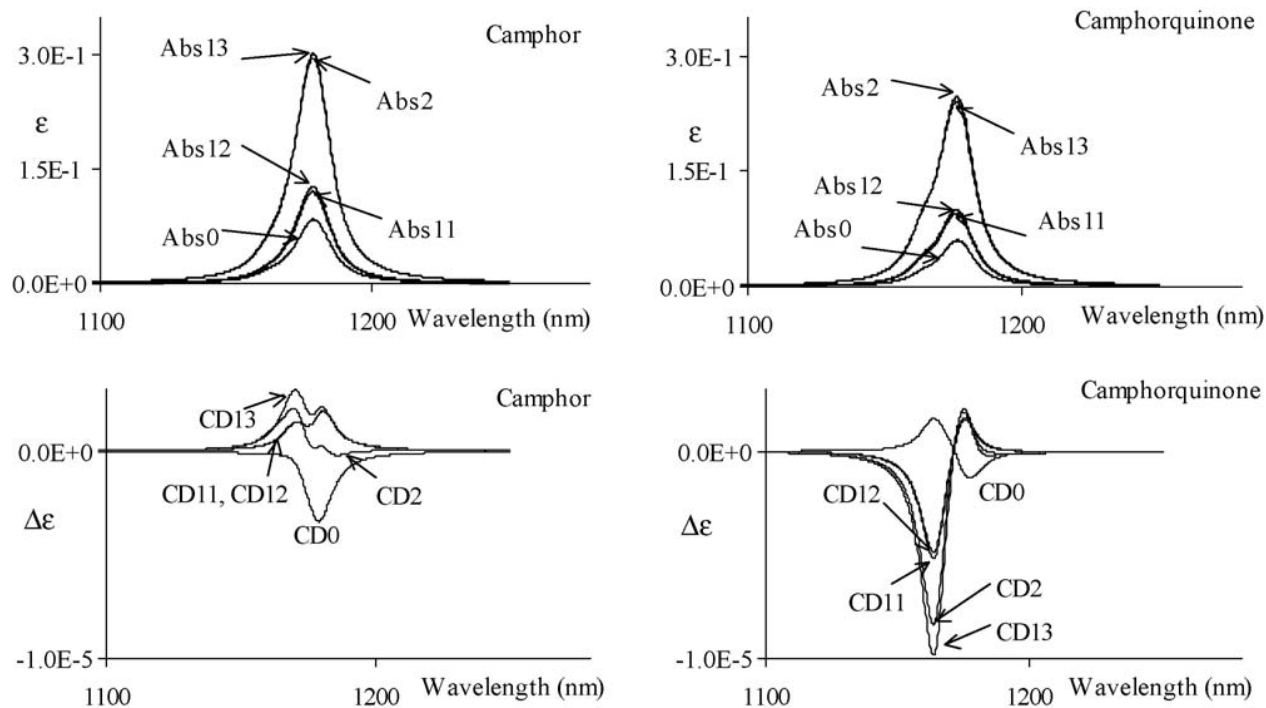

Fig. 5. Calculated absorption (top) and VCD (bottom) spectra for (1S)-Camphor (left) and (1S)-Camphorquinone (right) in the NIR region. The different curves correspond to different levels of approximation as described in the text. 
Table 2

Calculated wavelengths $\lambda(\mathrm{nm})$, dipole strengths D0, D13, D2 $\left(\times 10^{-42} \mathrm{esu}^{2} \mathrm{~cm}^{2}\right)$ and rotational strengths R0, R13, R2 $\left(\times 10^{-47}\right.$ esu' $\left.\mathrm{cm}^{2}\right)$ at various levels of approximation for (1S)-Camphor and (1S)-Camphorquinone (see text for discussion of orders of approximation).

\begin{tabular}{|c|c|c|c|c|c|c|c|}
\hline Local mode \# & $\lambda$ & D0 & D13 & D2 & R0 & R13 & $\mathrm{R} 2$ \\
\hline \multicolumn{8}{|l|}{ (1S)-Camphor } \\
\hline C7b-H & 1182.97 & 1.2321 & 3.4935 & 3.6460 & 1.4855 & -3.4283 & -4.5240 \\
\hline $\mathrm{C} 7 \mathrm{a}-\mathrm{H}$ & 1181.72 & 1.1997 & 3.4320 & 3.6619 & -4.9231 & -3.3889 & -3.5852 \\
\hline C6-H & 1181.15 & 0.9483 & 3.9490 & 3.5872 & 3.9803 & 11.1822 & 6.5542 \\
\hline $\mathrm{C} 5-\mathrm{H}$ & 1179.64 & 1.4717 & 4.5261 & 4.4411 & -4.3469 & 6.4693 & 3.8042 \\
\hline C6-H & 1179.31 & 0.9872 & 3.9956 & 3.8029 & -1.3605 & -3.6914 & -1.3044 \\
\hline C7b-H & 1178.65 & 1.0587 & 3.0020 & 3.2957 & -5.2300 & -4.2277 & -3.5365 \\
\hline $\mathrm{C} 3-\mathrm{H}$ & 1178.21 & 0.5755 & 4.0186 & 3.6807 & -5.7809 & 8.2278 & 8.4860 \\
\hline $\mathrm{C} 7 \mathrm{a}-\mathrm{H}$ & 1177.88 & 1.0065 & 2.9845 & 3.2690 & 6.1224 & 6.3929 & 6.2798 \\
\hline $\mathrm{C} 1 \mathrm{a}-\mathrm{H}$ & 1177.75 & 1.0130 & 2.7876 & 2.9146 & -2.8451 & -3.6134 & -5.3166 \\
\hline $\mathrm{C} 4-\mathrm{H}$ & 1177.05 & 1.6159 & 5.2522 & 4.4743 & -0.8630 & -4.5864 & -2.6421 \\
\hline C3-H & 1176.63 & 0.4351 & 3.7522 & 3.3084 & 5.0676 & -3.4637 & -3.6865 \\
\hline $\mathrm{C} 5-\mathrm{H}$ & 1176.25 & 1.3035 & 4.2641 & 4.0346 & -5.1971 & -4.6258 & -5.8813 \\
\hline $\mathrm{C} 1 \mathrm{a}-\mathrm{H}$ & 1174.50 & 0.8358 & 2.9165 & 3.1269 & -1.7169 & -0.0866 & -2.4789 \\
\hline $\mathrm{C} 1 \mathrm{a}-\mathrm{H}$ & 1171.83 & 0.6296 & 3.4066 & 3.4843 & 3.6184 & 8.0946 & 9.5427 \\
\hline $\mathrm{C} 7 \mathrm{a}-\mathrm{H}$ & 1168.96 & 0.7956 & 2.1939 & 2.6632 & 0.3407 & 5.3390 & 3.1666 \\
\hline $\mathrm{C} 7 \mathrm{~b}-\mathrm{H}$ & 1161.80 & 0.8781 & 2.2837 & 2.6222 & 0.3690 & -0.1579 & 1.5940 \\
\hline \multicolumn{8}{|c|}{ (1S)-Camphorquinone } \\
\hline C7a-H & 1179.48 & 0.7660 & 1.9475 & 2.6536 & -0.8957 & 0.3016 & 0.0114 \\
\hline $\mathrm{C} 7 \mathrm{~b}-\mathrm{H}$ & 1179.44 & 1.0071 & 3.3861 & 3.6117 & -0.1490 & -2.4337 & -5.2764 \\
\hline C6-H & 1178.00 & 0.8223 & 3.7617 & 3.5057 & 2.7342 & -0.3229 & -2.1827 \\
\hline $\mathrm{C} 6-\mathrm{H}$ & 1177.74 & 0.8317 & 3.8907 & 3.7457 & -0.5567 & -5.1379 & -2.3163 \\
\hline $\mathrm{C} 7 \mathrm{a}-\mathrm{H}$ & 1177.44 & 0.9179 & 3.3571 & 3.6057 & -2.8227 & -1.6210 & -0.4091 \\
\hline C7b-H & 1176.81 & 0.8848 & 3.0227 & 3.2390 & -4.2482 & 3.5935 & 7.2773 \\
\hline $\mathrm{C} 7 \mathrm{a}-\mathrm{H}$ & 1175.49 & 0.8622 & 2.9426 & 3.2599 & 4.8440 & 4.3354 & 4.0560 \\
\hline C5-H & 1175.30 & 0.8922 & 4.4011 & 4.2692 & 0.3622 & 11.5052 & 7.6426 \\
\hline $\mathrm{C} 1 \mathrm{a}-\mathrm{H}$ & 1175.11 & 0.8761 & 2.7918 & 2.8669 & -4.4660 & -1.5723 & -2.4015 \\
\hline $\mathrm{C} 5-\mathrm{H}$ & 1173.73 & 0.8430 & 4.3371 & 4.0284 & -3.1711 & 1.6500 & 1.5365 \\
\hline $\mathrm{C} 1 \mathrm{a}-\mathrm{H}$ & 1172.54 & 0.7134 & 2.8053 & 3.0170 & -1.7920 & -2.1202 & -3.9988 \\
\hline $\mathrm{C} 1 \mathrm{a}-\mathrm{H}$ & 1171.39 & 0.5572 & 3.3500 & 3.4621 & 4.6837 & 10.3443 & 12.5187 \\
\hline C7b-H & 1163.75 & 0.8339 & 2.3227 & 2.6944 & 1.4643 & -0.5377 & -0.5849 \\
\hline $\mathrm{C} 4-\mathrm{H}$ & 1163.66 & 0.6920 & 4.5616 & 3.7768 & 4.9762 & -42.4253 & -36.5177 \\
\hline
\end{tabular}

including all electric anharmonic terms. Finally, the isolated negative feature at lowest wavelength in the VCD spectrum of (1S)-Camphorquinone is due to the almost isolated $\mathrm{C}_{4} \mathrm{H}$ local mode.

In Fig. 6 the calculated spectra, obtained by including all corrections up to second-order, are compared with the experimental spectra. The agreement is rather satisfactory for absorption spectra of both molecules: the shape is predicted, also the shoulder at $1170 \mathrm{~nm}$ observed in Camphorquinone and not in Camphor absorption spectrum is reproduced; the total intensity is slightly overestimated and we think this to be related to the bandwidth, as mentioned above. We obtain a nice prediction for (1S)-Camphorquinone VCD spectrum, as well as for the overall intensity ratio between VCD spectra of (1S)-Camphor and (1S)-Camphorquinone. Some minor problems for the VCD of (1S)-Camphor are encountered. One should take into account that some of the approximations made here may have significant effects on the rather complex combination of rotational strengths of similar magnitude that give rise to the VCD spectrum of Camphor: besides the choice of the
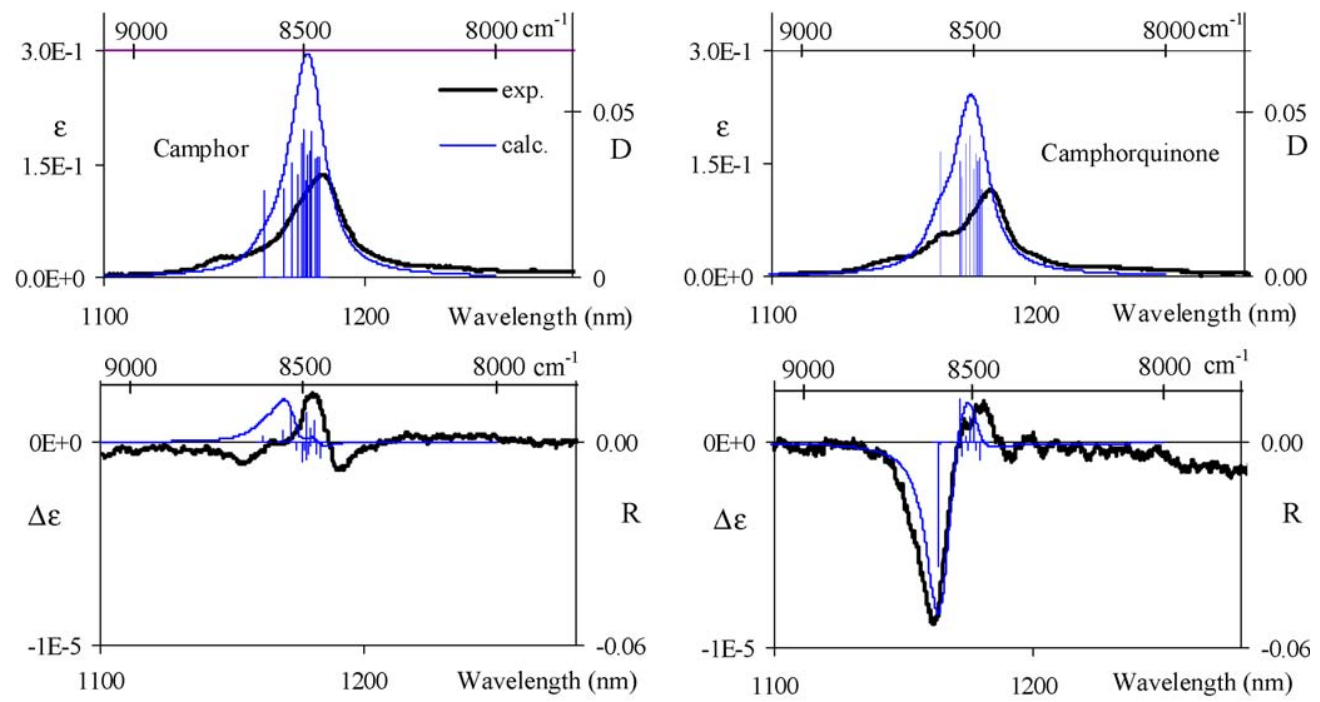

Fig. 6. Comparison of experimental and calculated NIR (top) and NIR-VCD (bottom) spectra of (1S)-Camphor (left) and (1S)-Camphorquinone (right) at $\Delta \nu=3$, with $\varepsilon$ and $\Delta \varepsilon$ in c.g.s. units. Vertical bars represent dipole strengths $D$ (right axis of top panels) in $10^{-40} \mathrm{esu}^{2} \mathrm{~cm}^{2}$ and rotational strengths $R$ (right axis of bottom panels) in $10^{-44}$ esu $\mathrm{cm}^{2}$. A wavenumber axis has been added at the top of each graph as in Fig. 1. The calculations are conducted following Bak et al. [21] as explained in the text. 
bandwidth, the frequency values and the anharmonicity parameter $\chi$ could be better estimated. The VCD spectrum of (1S)Camphorquinone seems to be less sensitive to such small corrections, because of the very strong negative band at low wavelengths that is almost entirely determined by few $\mathrm{CH}$ bond stretchings, $\mathrm{C}_{4} \mathrm{H}$ in particular.

\section{Conclusions}

The absorption and VCD spectra of (1S)-Camphor and (1S)Camphorquinone in the NIR region have been calculated in the local mode approximation, including electrical anharmonicity corrections and describing local $\mathrm{CH}$ stretchings by means of a Morse potential. $A b$ initio calculations and interpolation of the obtained numerical results have been performed to determine the first two derivatives of relevant APT and AAT components with respect to the internal stretching coordinate of each $\mathrm{CH}$ group.

The calculated absorption spectra satisfactorily reproduce the main features of the experimental ones for both molecules. The VCD spectrum of (1S)-Camphorquinone is also in very good agreement with experiment, while (1S)-Camphor is slightly less accurate, but still quite acceptable. In particular our analysis allows to point out the special role played by $\mathrm{C}_{4} \mathrm{H}$ which in Camphorquinone accounts for the evident shoulder at $1170 \mathrm{~nm}$ and for the most intense VCD signal. The latter VCD signal, whose importance has been noted in Ref. [1] for configurational analysis, is explained only by including anharmonic electric terms.

The procedure adopted here does not include the calculation of anharmonic force constants, and an experimentally determined value of the anharmonicity parameter $\chi=65 \mathrm{~cm}^{-1}$ has been applied to all $\mathrm{CH}$ bonds. An improvement of the results could be expected from a theoretical evaluation of this parameter. Efforts towards the determination of mechanical anharmonic terms have been made for several years $[30,31]$ and we will include their results in our future works.

The local-mode approach adopted here disregards also couplings between different bonds and this can affect our results. The molecules considered here have many $\mathrm{CH}$ bonds which are mechanically quite similar [2,3]: further developments should take into account mechanical and electrical couplings within methylene and methyl groups considering all mechanical and electrical anharmonic cross terms [26,27]. To implement and test this more general approach a smaller system containing fewer $\mathrm{CH}$-bond stretchings should be considered. In any case we reiterate that the prediction obtained on the basis of the strict local mode approximation is quite satisfactory for these large systems (16 inequivalent $\mathrm{CH}$ bonds): it reproduces the sign and intensity of the measured signals, the differences observed between the two molecules and it permits the assignment of the most intense VCD feature. Most importantly, the detailed analysis of the procedure adopted has allowed to point out the different relative importance of each electrical anarmonicity correction term in obtaining good prediction of experimental data.

With a view to future generalization, we notice that the expansion of the magnetic dipole moment here adopted is based on Ref. [21], but an additional term in $p^{3}$ is obtained at the same order in Ref. [17]. An analysis of the possible inclusion of such a contribution in the approach of Ref. [21] has not been considered in the present work. We notice that in Ref. [21] a discussion of the influence of non Born-Oppenheimer terms on anharmonic corrections to the absorption and VCD calculated spectra was provided and we think that discussion should be kept in mind in future progress of this work.

Finally, we wish to point out that this work is part of our ongoing programme for providing a fully non-parametric interpretation of the NIR-VCD spectra, comparable in quality to that used for the IR-VCD case, where just the harmonic approximation is needed. A little bit of additional work is still necessary for calculating anharmonic field corrections, anharmonic electrical interactions and, most important, from the knowledge of the full force field, appropriate evaluation of local mode mixing. We think we are close to achieving this goal and to report pretty soon results on this. Once this is achieved, anharmonic corrections can also be used to improve calculations of IR and VCD of fundamentals.

\section{Acknowledgements}

We wish to thank the Italian Ministry of Education, University and Research for help through the PRIN 2006 programme. We are also grateful to the Referee for helpful suggestions.

\section{Appendix A. Morse integrals}

In this appendix, matrix elements with Morse eigenfunctions [25] that have been used in the calculations described in the text are presented. Most of them are available in the literature [29,32]. Definitions and conventions here are essentially the same as in [32], and also the method to calculate new matrix elements is taken from that work.

The Morse potential has the form [25]:

$V(r)=D\left(1-\mathrm{e}^{-a\left(z-z_{e}\right)}\right)^{2}$

where $z$ is the distance between the two atoms and $z_{e}$ is its equilibrium value. The parameters $D$ and $a$ are related to the frequency $\omega$ and to the anharmonicity parameter $\chi$ (in wavenumbers) through the relations (31) and (32) in the text.

The eigenfunctions corresponding to the potential (A.1) are given by

$\psi_{v}(z)=N_{v} \mathrm{e}^{-\xi / 2} z^{b / 2} L_{v}^{b}(\xi)$

where $v$ is a non-negative integer, $L_{v}^{b}(\xi)$ is a Laguerre polynomial and [32]

$N_{v}=\left(\frac{a b v !}{\Gamma(k-v)}\right)^{1 / 2}$

$\xi=k \mathrm{e}^{-a\left(z-z_{e}\right)}$

$b=k-2 v-1$

$k=\frac{\omega}{\chi}$

In the calculations discussed in the text, matrix elements of some combinations of the operator $q=z-z_{e}$ and of its canonical conjugate $p$ are useful. Some results for transitions from the ground state to an excited one, some of which are the most common transitions in NIR absorption processes, and some are more useful for NIR-VCD, are reported below:

$$
\begin{aligned}
& \langle 0|q| v\rangle=\frac{A_{0 v}}{a} \frac{(-1)^{v+1}}{v(k-v-1)} \\
& \langle 0|p| v\rangle=\mathrm{i} a \frac{A_{0 v}}{2}(-1)^{v} \\
& \langle 0|q p| v\rangle=\mathrm{i} \frac{A_{0 v}}{2}(-1)^{v}\left(\log k+\frac{k-1}{v(k-v-1)}-\sum_{j=1}^{v} \frac{1}{j}-\psi(k-v)\right)
\end{aligned}
$$




$$
\begin{aligned}
\left\langle 0\left|q^{2}\right| v\right\rangle= & 2 \frac{A_{0 v}}{a^{2}} \frac{(-1)^{v+1}}{v(k-v-1)} \\
& \times\left(\log k-\sum_{j=1}^{v-1} \frac{1}{j}-\psi(k-v-1)\right) \\
\left\langle 0\left|q^{2} p\right| v\right\rangle= & 2 \frac{\log k}{a}\langle 0|q p| v\rangle-\left(\frac{\log k}{a}\right)^{2}\langle 0|p| v\rangle+\frac{\mathrm{i}}{a} \frac{N_{0} N_{v}}{a} \\
& \times\left(-\frac{1}{2} R_{1}^{0}+\frac{b}{2} R_{2}^{0}-R_{3}^{0}\right) \\
\left\langle 0\left|q^{3}\right| v\right\rangle= & \frac{N_{0} N_{v}}{a^{4}} \sum_{j=0}^{v}\left(\begin{array}{c}
v+b \\
v-j
\end{array}\right) \frac{(-1)^{j+1}}{j !} \Gamma(w)\left((\psi(w)-\log k)^{3}\right. \\
& \left.+3(\psi(w)-\log k) \psi^{(1)}(w)+\psi^{(2)}(w)\right)
\end{aligned}
$$

where the following notations have been used

$$
\begin{aligned}
A_{0 v}= & \frac{N_{0} N_{v}}{a} \Gamma(k-v) \\
R_{1}^{0}= & (-1)^{v} \Gamma(k \\
& -v)\left(2 \sum_{i=1}^{v} \sum_{j=i+1}^{v} \frac{1}{i j}+2 \sum_{i=1}^{v} \frac{1}{i} \psi(k-v)+\psi(k-v)^{2}+\zeta(2, k-v)\right) \\
R_{2}^{0}= & \frac{(-1)^{v}}{v} \Gamma(k-v-1)\left(\sum_{i=1}^{v-1} \frac{1}{i}+\psi(k-v-1)\right) \\
R_{3}^{0}= & (-1)^{v-1} \Gamma(k \\
& -v)\left(2 \sum_{i=1}^{v-1} \sum_{j=i+1}^{v-1} \frac{1}{i j}+2 \sum_{i=1}^{v-1} \frac{1}{i} \psi(k-v)+\psi(k-v)^{2}+\zeta(2, k-v)\right)
\end{aligned}
$$

$w=k-v+j-1$

and the special functions are as defined in [33], namely $\Gamma(z)$ in Section 8.31; $\psi(z)$ in Section 8.36; $\zeta(z, q)$ in Section 9.51. Moreover $\psi^{(n)}(z)=\mathrm{d}^{n} \psi(z) / \mathrm{d} z^{n}$.

Results (A.7), (A.8), (A.10) and (A.12) are special cases of those presented for example in [32] and in [29] for arbitrary initial state.

\section{References}

[1] E. Castiglioni, F. Lebon, G. Longhi, S. Abbate, Enantiomer 7 (2002) 161.
[2] G. Longhi, R. Gangemi, F. Lebon, E. Castiglioni, S. Abbate, V.M. Pultz, D.A. Lightner, J. Phys. Chem. A 108 (2004) 5338-5352.

[3] G. Longhi, S. Abbate, R. Gangemi, E. Giorgio, C. Rosini, J. Phys. Chem. A 110 (2006) 4958-4968.

[4] S. Abbate, E. Castiglioni, F. Gangemi, R. Gangemi, G. Longhi, R. Ruzziconi, S. Spizzichino, J. Phys. Chem. A 111 (2007) 7031-7040.

[5] L.A. Nafie, R.K. Dukor, R. Roy, A. Rilling, X. Cao, H. Buijs, Appl. Spectrosc. 57 (2003) 1245-1249.

[6] X. Cao, R.D. Shah, R.K. Dukor, C. Guo, T.B. Freedman, L.A. Nafie, Appl. Spectrosc. 58 (2004) 1057-1064

[7] C. Guo, R.D. Shah, R.K. Dukor, X. Cao, T.B. Freedman, L.A. Nafie, Appl. Spectrosc. 59 (2005) 1114-1124.

[8] C. Guo, X. Cao, T.B. Freedman, L.A. Nafie, Vibrational Spectrosc. 42 (2006) 254272.

[9] C. Guo, R.D. Shah, J. Mills, R.K. Dukor, X. Cao, T.B. Freedman, L.A. Nafie, Chirality 18 (2006) 775-782

[10] T.A. Keiderling, P.J. Stephens, Chem. Phys. Lett. 41 (1976) 46-49.

[11] (a) P.J. Stephens, F.J. Devlin, Chirality 12 (2000) 172;

b) P.J. Stephens, Encyclopaedia of Spectroscopy and Spectrometry, Academic Press, London, 1999, p2415.

[12] M.S. Child, L. Halonen, Adv. Chem. Phys. LVII (1985) 1-58.

[13] B.R. Henry, Acc. Chem. Res. 10 (1987) 429-435.

[14] S. Abbate, R. Gangemi, G. Longhi, J. Chem. Phys. 117 (2002) 7575-7586.

15] R. Gangemi, G. Longhi, S. Abbate, Chirality 17 (2005) 530-539.

[16] M.J. Frisch, G.W. Trucks, H.B. Schlegel, G.E. Scuseria, M.A. Robb, J.R. Cheeseman, J.A. Montgomery Jr., T. Vreven, K.N. Kudin, J.C. Burant, J.M. Millam, S.S. Iyengar, J. Tomasi, V. Barone, B. Mennucci, M. Cossi, G. Scalmani, N. Rega, G.A. Petersson, H. Nakatsuji, M. Hada, M. Ehara, K. Toyota, R. Fukuda, J. Hasegawa, M. Ishida, T. Nakajima, Y. Honda, O. Kitao, H. Nakai, M. Klene, X. Li, J.E. Knox, H.P. Hratchian, J.B. Cross, C. Adamo, J. Jaramillo, R. Gomperts, R.E. Stratmann, O. Yazyev, A.J. Austin, R. Cammi, C. Pomelli, J.W. Ochterski, P.Y. Ayala, K. Morokuma, G.A. Voth, P. Salvador, J.J. Dannenberg, V.G. Zakrzewski, S. Dapprich, A.D. Daniels, M.C. Strain, O. Farkas, D.K. Malick, A.D. Rabuck, K. Raghavachari, J.B. Foresman, J.V. Ortiz, Q. Cui, A.G. Baboul, S. Clifford, J. Cioslowski, B.B. Stefanov, G. Liu, A. Liashenko, P. Piskorz, I. Komaromi, R.L. Martin, D.J. Fox, T. Keith, M.A. Al-Laham, C.Y. Peng, A. Nanayakkara, M. Challacombe, P.M.W. Gill, B. Johnson, W. Chen, M.W. Wong, C. Gonzalez, J.A. Pople, Gaussian 03, Revision B.05, Gaussian, Inc., Pittsburgh, PA, 2003.

[17] P.L. Polavarapu, Mol Phys. 89 (1996) 1503-1510.

[18] S. Abbate, G. Longhi, C. Santina, Chirality 12 (2000) 180-190.

[19] P.J. Stephens, J. Phys. Chem. 89 (1985) 748-750.

[20] (a) A.D. Buckingham, P.W. Fowler, P.A. Galwas, Chem. Phys. 112 (1987) 1 (b) PA. Galwas, Ph.D. Thesis, Cambridge University, Cambridge, UK, 1983.

[21] K.L. Bak, O. Bludský, P. Jørgensen, J. Chem. Phys. 103 (1995) 10548-10555.

[22] L.Z. Rosenfeld, Physik 52 (1928-29) 16.

[23] (a) M. Born, J.R. Oppenheimer, Ann. Phys. 84 (1927) 457-484; (b) A. Mead, A. Moscowitz, Int. J. Quant. Chem. 1 (1981) 243.

[24] J.R. Cheeseman, F.J. Devlin, M.J. Frisch, P.J. Stephens, Chem. Phys. Lett. 252 (1996) 211.

[25] (a) P.M. Morse, Phys. Rev. 34 (1929) 57; (b) D. Ter Haar, Phys. Rev. 70 (1946) 222.

[26] H.G. Kjaergaard, B.R. Henry, J. Chem. Phys. 96 (1992) 4841-4851.

[27] D.M. Turnbull, H.G. Kjaergaard, B.R. Henry, Chem. Phys. 195 (1995) 129-141.

[28] G. Longhi, G. Zerbi, L. Ricard, S. Abbate, J. Chem. Phys. 88 (1988) 67336741.

[29] M.L. Sage, Chem. Phys. 35 (1978) 375-380

[30] R. Burcl, N.C. Handy, S. Carter, Spectrochim. Acta 59 (2003) 1881-1893.

[31] V. Barone, J. Chem. Phys. 122 (2005) 14108.

[32] J.A.C. Gallas, Phys. Rev. A 21 (1980) 1829-1834.

[33] I.S. Gradshteyn, I.M. Ryzhik, Table of Integrals, Series, and Products, Academic Press, New York, 1980 